\title{
ВЛИЯНИЕ ВНУТРИСКВАЖИННОГО КОМПЕНСАТОРА НА ПАДЕНИЕ НАПРЯЖЕНИЯ В ЭЛЕМЕНТАХ ЭЛЕКТРОТЕХНИЧЕСКОГО КОМПЛЕКСА ДОБЫВАЮЩЕЙ СКВАЖИНЫ
}

\author{
Копырин Владимир Анатольевич', \\ kopyrinva@gmail.com \\ Смирнов Олег Владимирович', \\ oleg_smirnov_1940@mail.ru \\ Портнягин Алексей Леонидович', \\ pal1979@mail.ru \\ Хамитов Рустам Нуриманович², \\ apple_27@mail.ru \\ 1 Тюменский индустриальный университет, \\ Россия, 625000, г. Тюмень, ул. Володарского, 38. \\ 2 Омский государственный технический университет, \\ Россия, 644050, г. Омск, пр. Мира, 11.
}

\begin{abstract}
Актуальность исследования обусловлена необходимостью определения требуемого напряжения на выходе питающего трансформатора с учетом компенсации реактивной мощности внутри скважины, с целью предотвращения аварийных ситуаций в процессе эксплуатации установок электроцентробежных насосов.

Цель исследования: оценить изменение величины падения напряжения в токопроводящих элементах электротехнического комплекса установки электроцентробежного насоса для добычи нефти при использовании внутрискважинного компенсатора реактивной мощности.

Объект исследования: электротехнический комплекс установок электроцентробежных насосов для добычи нефти.

Методы. При разработке математической модели электротехнического комплекса погружной установки для добычи нефти использованы положения теоретических основ электротехники, корректные допущения при составлении математических моделей и компьютерное моделирование в программном пакете символьной математики Wolfram Mathematica.

Результаты. Разработана схема замещения и математическая модель электротехнического комплекса установки электроцентробежных насосов с внутрискважинным компенсатором реактивной мощности. С помощью качественной векторной диаграммы напряжений и токов в ветвях и узлах схемы замещения показано распределение величин падений напряжений на элементах комплекса. Анализ векторной диаграммы показал, что внутрискважинный компенсатор уменьшает продольную и поперечную составляющие падения напряжения, что положительно влияет на режим работы установки для добычи нефти. Получены поверхности требуемого напряжения на выходе питающего трансформатора в зависимости от мощности погружного электродвигателя для различных длин и сечений кабельной линии. Установлено, что использование внутрискважинного компенсатора позволяет уменьшить падение напряжения в токопроводящих элементах электротехнического комплекса установки для добычи нефти до 50 B.
\end{abstract}

\section{Ключевые слова:}

Внутрискважинный компенсатор, напряжение отпайки трансформатора, падение напряжения, реактивная мощность, схема замещения.

\section{Введение}

Повышение энергетической эффективности производства является первостепенной задачей, стоящей перед научно-техническим сообществом, решение которой способствует повышению энергетической безопасности Российской Федерации. В настоящее время снижение доли потерь от суммарной потребляемой энергии достигается модернизацией существующего оборудования с учетом специфических особенностей условий эксплуатаций, внедрением новых более эффективных технологических решений, а также внедрением организационных мероприятий.

В России действующий эксплуатационный фонд нефтяных скважин составляет порядка 135 тыс. единиц, из которых свыше 60 \% оборудованы установками электроцентробежных насо- сов (УЭЦН) [1-3] и их доля продолжает увеличиваться.

Известно, что коэффициент полезного действия УЭЦН не превышает $30 \%$ [4, 5]. Основная доля потерь энергии до 43 \% при эксплуатации установок электроцентробежных насосов приходится на электротехнический комплекс (ЭТК) для добычи нефти. В связи с этим актуальными становятся вопросы повышения энергетической эффективности и обеспечения энергосбережения электротехнических комплексов погружных установок для добычи нефти.

Вопросами компенсации реактивной мощности в электроэнергетических системах с целью регулирования напряжения, повышения качества электроэнергии, снижения потерь электроэнергии занимались многие отечественные и зарубежные 
ученые: Б.Н. Абрамович [6, 7], И.В. Жежеленко [8], Ю.С. Железко [9], Н. Akagi [10], L. Czarnecki $[11,12]$, D. Sharon $[13,14]$ и другие. В указанных работах авторами предложены методики определения реактивной мощности, описаны и предложены устройства компенсации, способы управления данными устройствами, доказана эффективность их применения.

Для повышения эффективности использования электроэнергии ЭТК УЭЦН в работах [15-17] предложено применять внутрискважинные компенсаторы реактивной мощности (ВКРМ), которые можно разделить на две группы: регулируемые и нерегулируемые. Авторами установлено, что внедрение технологии внутрискважинной компенсации реактивной мощности на нефтедобывающих объектах позволяет повысить энергетическую эффективность добычи нефти до 15,8 \% . Суть технологии заключается в том, что компенсирующее устройство устанавливается непосредственно в скважине и подключается параллельно зажимам погружного электродвигателя. Повышение энергетической эффективности достигается за счет уменьшения потерь активной мощности в токопроводящих элементах электротехнического комплекса вследствие снижения потребляемого тока.

Тем не менее, если при эксплуатации нефтяных скважин, оборудованных УЭЦН, не учитывать тот факт, что использование внутрискважинных компенсаторов приводит к снижению падения напряжения в узлах электротехнического комплекса, за счет уменьшения потребляемого тока [18-20], может произойти случай, когда напряжение на погружном электродвигателе и самом компенсаторе будет выше заданного значения при неправильном выборе отпайки питающего трансформатора. Это может привести к снижению ресурса погружного электрооборудования и нарушению технологии добычи. Для батарей конденсаторов, чувствительных к повышению напряжения [18], в некоторых случаях допускается превышение уровня напряжения на $10 \%$ выше номинального продолжительностью не более 8 часов.

В настоящее время поддержание напряжения близкого к номинальному на погружном электродвигателе достигается изменением отпайки питающего трансформатора или изменением напряжения на выходе станции управления, оборудованной преобразователем частоты. Существующие технические регламенты большинства нефтедобывающих компании по ремонту, обслуживанию и выводу на режим скважин с УЭЦН составлены без учета компенсации реактивной мощности внутри скважины. Поэтому данная статья имеет практический интерес для инженеров и энергетиков нефтедобывающих и сервисных компаний при корректировке технических регламентов в части определения напряжения на выходе питающего трансформатора.

\section{Объект и методы исследования}

Для оценки влияния внутрискважинного компенсатора на величину падения напряжения на элементах электротехнического комплекса УЭЦН, который включает: источник питания, питающий трансформатор, кабельную линию (КЛ), погружной электродвигатель и внутрискважинный компенсатор реактивной мощности, разработана и приведена на рис. 1 схема замещения комплекса. Согласно рекомендациям положений теоретических основ электротехники для симметричных трехфазных систем, к которым относится ЭТК УЭЦН, моделирование производится для одной фазы.

Схема замещения ЭТК УЭЦН (рис. 1) по методу узловых потенциалов описывается системой матричных уравнений:

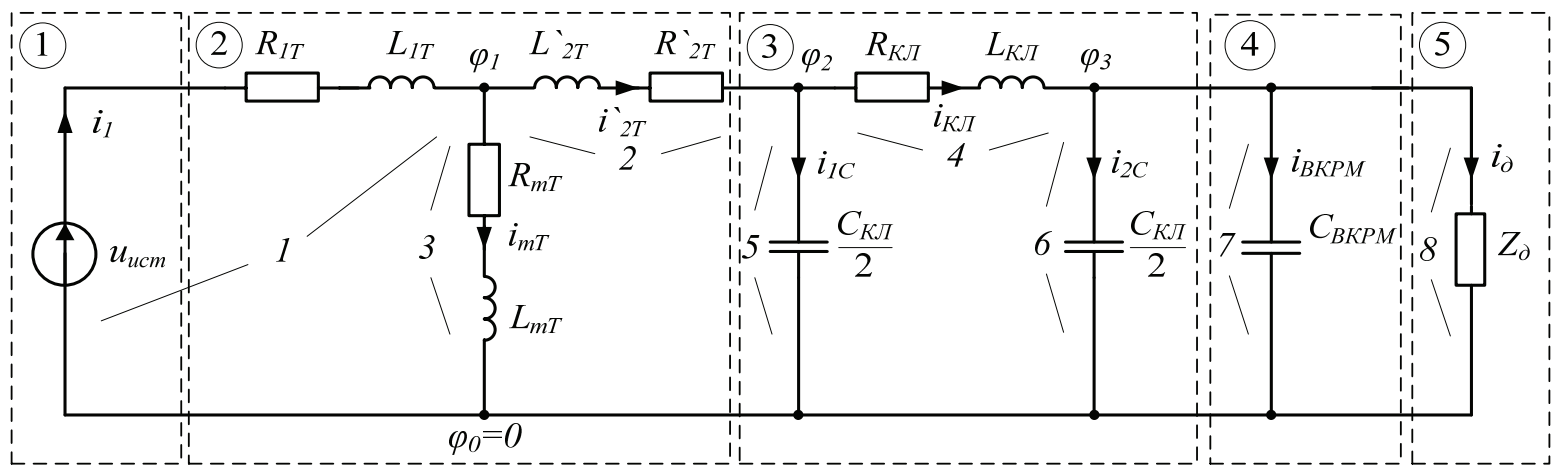

Pис. 1. Схема замещения ЭТК УЭЦН: 1 - источник питания; 2 - питающий трансформатор; 3 - кабельная линия; 4 - внутрискважинный компенсатор реактивной мощности; 5 - погружной асинхронный электродвигатель; $u_{\text {ис, }} i_{\text {ист }}$ - напряжение и ток источника питания; Z, R, L, C - полное и активное сопротивления, индуктивность и емкость соответствующих элементов; $\varphi, i$ - потенциалы узлов и токи ветвей

Fig. 1. Equivalent circuit of the ETC ESP: 1 is the power source; 2 is the power supply transformer; 3 is the cable; 4 is the downhole reactive power compensator; 5 is the submersible induction motor; $u_{5 y s}, i_{5 y s}$ is the voltage and current power source; $Z, R, L, C$ is the full and active resistance, inductance, and capacitance of the respective elements; $\varphi$, $i$ are the the nodes' potentials and the branch currents 


$$
\left[\begin{array}{c}
\left(Y_{1}+Y_{2}+Y_{3}\right) \varphi_{1}-Y_{2} \varphi_{2} \\
-Y_{2} \varphi_{1}+\left(Y_{2}+Y_{4}+Y_{5}\right) \varphi_{2}-Y_{4} \varphi_{3} \\
-Y_{4} \varphi_{2}+\left(Y_{4}+Y_{6}+Y_{7}+Y_{8}\right) \varphi_{3}
\end{array}\right]=\left[\begin{array}{c}
\dot{U}_{\text {ист }} \\
0 \\
0
\end{array}\right],
$$

где $Y$ - комплексные проводимости соответствующих ветвей.

Напряжения и токи соответствующих узлов и ветвей схемы замещения определяются следующим образом:

$$
\dot{U}_{i}=\left[\begin{array}{c}
-\varphi_{1} \\
\varphi_{1}-\varphi_{2} \\
\varphi_{1} \\
\varphi_{2}-\varphi_{3} \\
\varphi_{2} \\
\varphi_{3} \\
\varphi_{3} \\
\varphi_{3}
\end{array}\right], \quad \dot{I}_{i}=\left[\begin{array}{c}
Y_{1}\left(\dot{U}_{\text {ист }}-\varphi_{1}\right) \\
Y_{2}\left(\varphi_{1}-\varphi_{2}\right) \\
\mathrm{Y}_{3} \varphi_{1} \\
Y_{4}\left(\varphi_{2}-\varphi_{3}\right) \\
\mathrm{Y}_{5} \varphi_{2} \\
\mathrm{Y}_{6} \varphi_{3} \\
\mathrm{Y}_{7} \varphi_{3} \\
\mathrm{Y}_{8} \varphi_{3}
\end{array}\right],
$$

где $\dot{U}_{i}, \dot{I}_{i}-$ напряжение и ток на $i$ узле, ветви соответственно, $i=1 . . .8$.

Для качественного анализа влияния внутрискважинного компенсатора на электрические параметры ЭТК УЭЦН построим в соответствии с выражениями (1) и (2) векторную диаграмму напряжений и токов (рис. 2) в узлах и ветвях схемы. Масштабы векторов напряжений и токов на векторной диаграмме могут не совпадать. Для удобства построения за начальный вектор принято напряжение на погружном электродвигателе.

На диаграмме видно, что ток $\dot{I}_{\text {кл }}$, протекающий по кабельной линии, представляет собой геометрическую сумму токов погружного электродвигателя

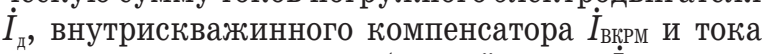
проводимости в конце кабельной линии $\dot{I}_{2 \mathrm{C}}$.

Для определения падения напряжения на кабельной линии $\Delta \dot{U}_{32}$ откладываем от конца вектора напряжения на погружном электродвигателе $\dot{U}_{\text {д }}$ параллельно вектору тока $\dot{I}_{\text {кл }}$ продольную составляющую падения напряжения на активном со-

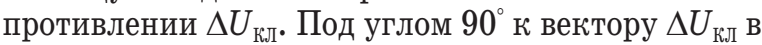

сторону опережения откладываем поперечную составляющую падения напряжения на реактивном

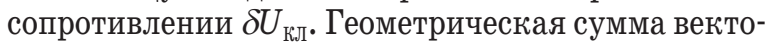
ров $\Delta U_{\text {кл }}$ и $\delta U_{\text {кл }}$ равна падению напряжения в кабельной линии $\Delta \dot{U}_{32}$.

Емкостной ток проводимости в конце КЛ $\dot{I}_{2 \mathrm{C}}$ и емкостной ток внутрискважинного компенсатора $\dot{I}_{\text {Вкрм }}$, протекая по линии совместно с током погружного электродвигателя $\dot{I}_{д}$, компенсируют соответствующую величину индуктивной составляющей погружного электродвигателя.

Чтобы определить ток $\dot{I}_{1}$, потребляемый из се-

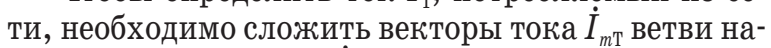
магничивания и тока $\dot{I}_{2 \mathrm{~T}}^{\prime}$, протекающего по вторичной обмотке трансформатора. Ток $\dot{I}_{2 \mathrm{~T}}^{\prime}$ представляет собой геометрическую сумму вектора тока кабельной линии $\dot{I}_{\text {кл }}$ и тока проводимости в начале кабельной линии $\dot{I}_{1 \mathrm{c}}$.

Для определения падения напряжения на вторичной обмотке трансформатора $\Delta \dot{U}_{21}$ от конца вектора $\Delta \dot{U}_{32}$ параллельно вектору тока $\dot{I}_{2 \mathrm{~T}}^{\prime}$ откладываем продольную составляющую падения напряжения на активном сопротивлении $\Delta U_{2 T}$. Под углом $90^{\circ} \mathrm{\kappa}$ вектору $\Delta U_{2 \mathrm{~T}}$ в сторону опережения откладываем поперечную составляющую падения напряжения на реактивном сопротивлении $\delta U_{2 T}$. Геометрическая сумма векторов $\Delta U_{2 T}$ и $\delta U_{2 T}$ равна падению напряжения на вторичной обмотке трансформатора $\Delta \dot{U}_{21}$.

Для определения падения напряжения на первичной обмотке трансформатора $\Delta \dot{U}_{1 \mathrm{~T}}$ вектор падения напряжения на активном сопротивлении следует откладывать параллельно вектору тока $\dot{I}_{1}$, аналогично вторичной обмотке.

Геометрическая сумма векторов $\dot{U}_{\mathbb{д}}, \Delta \dot{U}_{32}, \Delta \dot{U}_{21}$ и $\Delta \dot{U}_{1 \mathrm{~T}}$ составляет напряжение источника питания.

Из диаграммы следует, что внутрискважинный компенсатор положительно влияет на режим работы ЭТК УЭЦН. Увеличение поперечной емкостной составляющей в конце кабельной линии приводит к снижению токов в кабельной линии и обмотках питающего трансформатора и как следствие уменьшению падения напряжения в элементах ком-

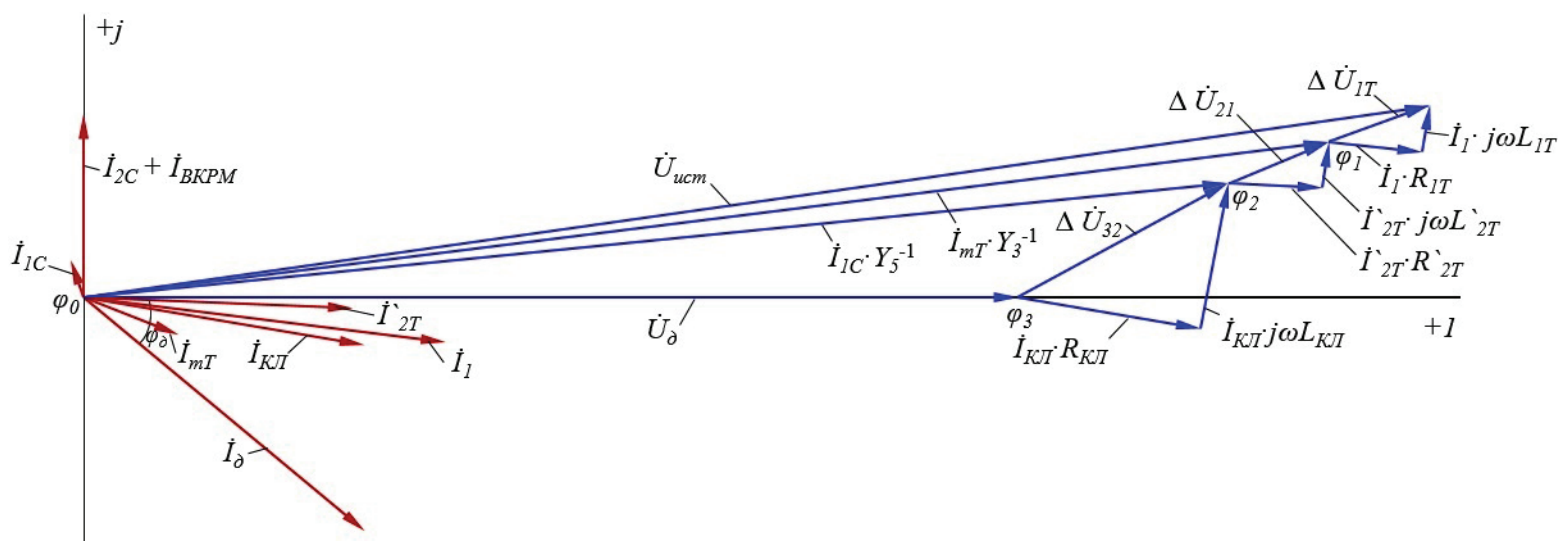

Pис. 2. Качественная векторная диаграмма ЭТК УЭЦН

Fig. 2. Qualitative vector diagram ETC ESP 
плекса. Тем не менее снижение падения напряжения приводит к повышению напряжения на погружном электродвигателе и внутрискважинном компенсаторе. Это может привести к перекомпенсации и нивелированию положительного эффекта. Поэтому напряжение отпайки трансформатора следует задавать с учетом внутрискважинного компенсатора.

Требуемое напряжение на выходе питающего трансформатора, согласно схеме замещения (рис. 1) и векторной диаграмме (рис. 2), определяется по выражению:

$$
U_{\text {тмпн }}=\left|\dot{U}_{\text {д.ном }}+\Delta \dot{U}_{\mathrm{T}}+\Delta \dot{U}_{\text {Кл }}\right|,
$$

где $\dot{U}_{\text {д.ном }}$ - комплексное номинальное напряжение погружного электродвигателя; $\Delta \dot{U}_{T}-$ падение напряжения в обмотках трансформатора; $\Delta \dot{U}_{\mathrm{k}}-$ падение напряжения в кабельной линии.

Падение напряжения в обмотках трансформатора без учета ветви намагничивания, ввиду его незначительного влияния, определяется по выражению:

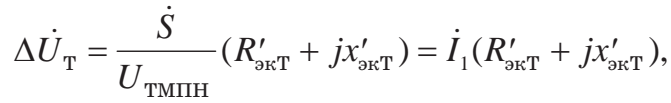

где $\dot{S}$ - комплексная полная мощность, потребляемая УЭЦН; $U_{\text {тмпн }}-$ напряжение на выходе питающего трансформатора; $\dot{I}_{1}$ - ток сети, $\dot{I}_{1}=\dot{I}_{2 \mathrm{~T}}^{\prime} ; R_{\text {экТ }}^{\prime}$, $X_{\text {экт }}^{\prime}$ - приведенные эквивалентные активное и реактивное сопротивления питающего трансформатора.

Приведенные эквивалентные активное и реактивное сопротивления питающего трансформатора определяются по известным выражениям:

$$
\begin{aligned}
& R_{\text {экт }}^{\prime}=R_{1 \mathrm{~T}}+R_{2 \mathrm{~T}}^{\prime}=\frac{\Delta P_{\mathrm{K}} U_{\text {вн.ном }}^{2} \cdot 10^{3}}{S_{\text {вн.ном }}^{2}} ; \\
& x_{\text {экТ }}^{\prime}=x_{1 \mathrm{~T}}+x_{2 \mathrm{~T}}^{\prime}=\frac{u_{\mathrm{K}} \% U_{\text {вн.ном }}^{2} \cdot 10^{3}}{100 S_{\text {ном }}},
\end{aligned}
$$

где $\Delta P_{\mathrm{k}}-$ потери короткого замыкания, кВт; $U_{\text {вн.но }}-$ номинальное напряжение высшей ступени трансформатора, кВ; $S_{\text {ном }}$ - полная мощность трансформатора, кВА; $u_{\mathrm{k}} \%$ - напряжение короткого замыкания, \% .

Падение напряжения в кабельной линии определяется по выражению:

$$
\Delta \dot{U}_{\text {кл }}=\dot{I}_{1}\left(R_{\text {кл }}+j x_{\text {кл }}\right),
$$

где $R_{\text {Кл }}, X_{\text {Кл }}$ - активное и реактивное сопротивления кабельной линии, которые определяются по выражениям:

$$
\begin{gathered}
R_{\text {кл }}=10^{3} \rho\left(\frac{l_{1}}{S_{1}}+\frac{l_{2}}{S_{2}}\right)[1+0,004(T-20)] ; \\
x_{\text {кл }}=x_{\text {о }}\left(l_{1}+l_{2}\right),
\end{gathered}
$$

где $\rho$ - удельное сопротивление проводника, $\mathrm{OM} \cdot \mathrm{MM}^{2} / \mathrm{m} ; l_{1}, l_{2}$ - длина основного кабеля и кабеляудлинителя соответственно, км; $S_{1}, S_{2}$ - сечение токопроводящей жилы основного кабеля и кабеля-уд- линителя соответственно, $\mathrm{MM}^{2} ;$ T - температура токопроводящих жил кабелей, ${ }^{\circ} \mathrm{C} ; x_{0}-$ погонное индуктивное сопротивление кабельной линии, Ом/км.

В настоящее время с увеличением доли трудноизвлекаемых запасов углеводородов длина кабельной линии нередко составляет 2000 м, а в некоторых случаях достигает 3500 м. Температуры пластовых жидкостей на таких глубинах варьируются от 40 до $120{ }^{\circ} \mathrm{C}$ и могут повышаться до $150{ }^{\circ} \mathrm{C}$ $[21,22]$. В связи с этим расчетная температура для определения активного сопротивления токопроводящих жил кабельной линии принимается $70{ }^{\circ} \mathrm{C}$.

В качестве питающего кабеля, как правило, используется кабель марки КПБП или КПБК плоского или круглого сечения с изоляцией из фторопласта. Если погонная емкость данных кабелей составляет 0,1 мк $\Phi / к м$, а емкостное сопротивление при частоте 50 Гц равно 31,847 кОм/км и на порядок выше активного и индуктивного сопротивления, то ее можно исключить из схемы замещения (рис. 1) и не учитывать при расчетах. Принятое допущение не сильно повлияет на точность определения падения напряжения в элементах ЭТК УЭЦН.

Тогда ток сети, с учетом принятых допущений, согласно I-му закону Кирхгофа определяется по выражению:

$$
\dot{I}_{1}=\dot{I}_{\mathrm{BKPM}}+\dot{I}_{\text {д }} \text {. }
$$

Согласно схеме замещения (рис. 1), напряжение на внутрискважинном компенсаторе равно напряжению на погружном электродвигателе. Токи, потребляемые внутрискважинным компенсатором и погружным электродвигателем, определяются по выражениям:

$$
\begin{gathered}
\dot{I}_{\text {вКРм }}=\frac{j Q_{\text {ВКРМ }}}{\sqrt{3} \dot{U}_{\text {д.ном }}} ; \\
\dot{I}_{\text {д }}=\frac{\tilde{S}_{\text {д.ном }}}{\sqrt{3} \dot{U}_{\text {д.ном }}}=\frac{P_{\text {д.ном }}-j Q_{\text {д.ном }}}{\sqrt{3} \dot{U}_{\text {д.ном }}},
\end{gathered}
$$

где $Q_{\text {вкрм }}$ - реактивная мощность внутрискважинного компенсатора, вар; $\widetilde{S}_{\text {д.ном }}, P_{\text {д.ном }}, Q_{\text {д.ном }}-$ комплексно-сопряженная полная, активная и реактивная мощности, потребляемые погружным электродвигателем соответственно, ВА, Вт, вар.

Преобразуем выражение (3) согласно (4)-(8):

$$
=\left|\begin{array}{c}
U_{\text {тмпн }}= \\
\dot{U}_{\text {д.ном }}+\left(\frac{P_{\text {д.ном }}}{\sqrt{3} \dot{U}_{\text {д.ном }}}+\frac{j Q_{\mathrm{BKPM}}-j Q_{\text {д.ном }}}{\sqrt{3} \dot{U}_{\text {д.ном }}}\right) \times \mid \\
\times\left(Z_{\text {экт }}^{\prime}+Z_{\text {Кл }}\right)
\end{array}\right|,
$$

где $Z_{\text {экт }}^{\prime}, Z_{\text {кл }}$ - комплексные полные сопротивления питающего трансформатора и кабельной линии соответственно.

Полученное выражение позволяет определить требуемое напряжение на выходе питающего трансформатора с учетом внутрискважинного компенсатора реактивной мощности и подобрать отпайку трансформатора. 


\section{Результаты моделирования}

Для оценки влияния внутрискважинного компенсатора на величину падения напряжения в элементах электротехнического комплекса рассмотрим УЭЦН, оснащенную трансформатором марки ТМПН 400/3. Расчетное полное эквивалентное сопротивление трансформатора равно [16]:

$$
Z_{\text {экт }}^{\prime}=0,23+j 0,859 \text { Oм. }
$$

Диапазон изменения мощностей погружного электродвигателя принимается от 100 до 250 кВт. Коэффициент мощности погружных электродвигателей принимается 0,735 [16]. Требуемый уровень коэффициента мощности сети в точке подключения внутрискважинного компенсатора принимается равным 0,95 .

C помощью специализированного программного пакета символьной математики Wolfram Mathematica получены и приведены на рис. 3 результаты расчета требуемого уровня напряжения отпайки трансформатора в зависимости от мощности погружного электродвигателя, длин и сечений кабельной линии.

Анализ поверхностей (рис. 3) показал, что использование внутрискважинного компенсатора приводит к снижению требуемого уровня напряжения отпайки питающего трансформатора во всех диапазонах мощностей погружных электро- двигателей, длин и сечений кабельной линии. Например, для УЭЦН, оснащенной погружным электродвигателем мощностью $200 \mathrm{\kappa B}$, кабельной линией длиной $1500 \mathrm{~m}$, сечением токопроводящих жил 16 мм² $^{2}$, требуемое напряжение отпайки трансформатора до компенсации реактивной мощности внутри скважины составило 2667 В, после компенсации - 2635 В.

Таким образом, использование внутрискважинных компенсаторов в составе установок электроцентробежных насосов на нефтедобывающих объектах приводит к снижению падения напряжения, что положительно сказывается на режиме работы установки.

\section{Заключение}

1. Получена схема замещения и математическая модель электротехнического комплекса УЭЦН. На основании разработанной схемы замещения и математической модели построена качественная векторная диаграмма ЭТК УЭЦН, анализ которой показал, что использование внутрискважинного компенсатора реактивной мощности приводит к снижению падения напряжения в элементах комплекса.

2. Получены поверхности требуемого уровня напряжения отпайки трансформатора до и после
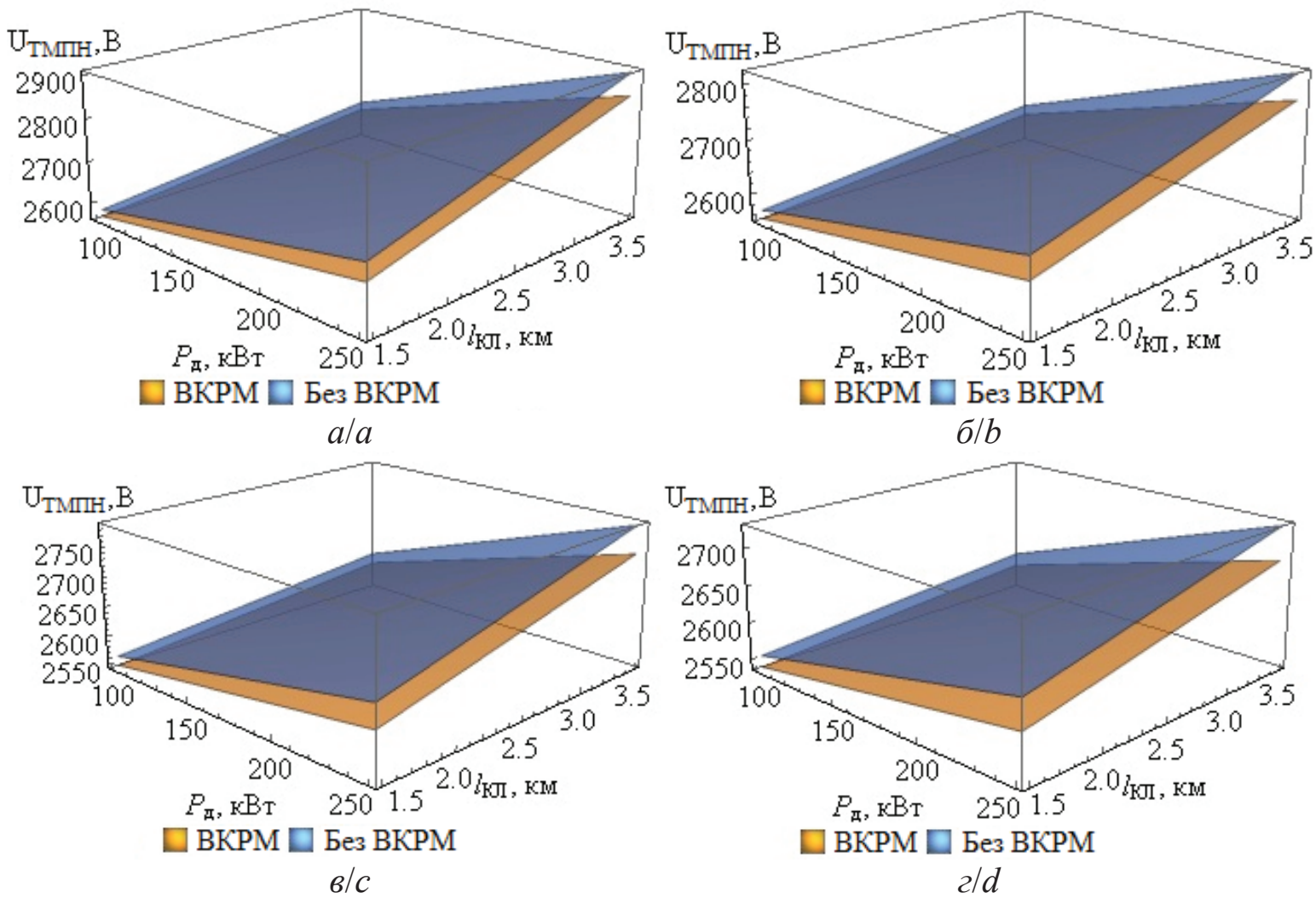

Pис. 3. Поверхности требуемого уровня напряжения отпайки трансформатора до и после компенсации реактивной мощности внутри скважины при $U_{\text {дном }}=2500$ B: a) $S_{K л}=16 \mathrm{MM}^{2}$; б) $S_{K л}=21,15 \mathrm{Mm}^{2}$; в) $S_{K л}=25 \mathrm{MM}^{2}$; г) $S_{K л}=35 \mathrm{MM}^{2}$

Fig. 3. Surface of the required voltage level of the transformer before and after compensation of reactive power inside the well at $U_{\text {m.nom }}=2500 \mathrm{~V}$ : a) $S_{C L}=16 \mathrm{~mm}^{2}$; b) $S_{C L}=21,15 \mathrm{~mm}^{2}$; c) $S_{C L}=25 \mathrm{~mm}^{2}$; d) $S_{C L}=35 \mathrm{~mm}^{2}$ 
компенсации реактивной мощности внутри скважины. Установлено, что использование внутрискважинных компенсаторов позволяет уменьшить падение напряжение в элементах электротехнического комплекса УЭЦН до $50 \mathrm{~B}$.

\section{СПИСОК ЛИТЕРАТУРЫ}

1. Вопросы энергоэффективности установок электроприводных центробежных насосов / В.Н. Ивановский, А.А. Сабиров, А.В. Деговцов, Ю.А. Донской, А.В. Булат, А.С. Зуев, С.Б. Якимов // Оборудование и технологии для нефтегазового комплекса. - 2016. - № 4. - С. 25-30.

2. Боловин Е.В., Глазырин А.С. Метод идентификации параметров погружных асинхронных электродвигателей установок электроприводных центробежных насосов для добычи нефрти // Известия Томского политехнического университета. Инжиниринг георесурсов. - 2017. - Т. 328. - № 1. - С. 123-131.

3. Букреев В.Г., Сипайлова Н.Ю., Сипайлов В.А. Стратегия управления электротехническим комплексом механизированной добычи нефти на основе экономического критерия // Известия Томского политехнического университета. Инжиниринг георесурсов. - 2017. - Т. 328. - № 3. - С. $75-84$.

4. Невоструев В.А. Комплексный подход к энергоэффективности при добыче нефти УЭЦН // Инженерная практика. - 2017. № 8. - C. 28-32.

5. Якимов С.Б., Каверин М.Н., Тарасов В.П. 0 новых перспективах применения ПЭД с повышенным напряжением питания для снижения капитальных и операционных затрат // 0борудование и технологии для нефтегазового комплекса. - 2015. № 4. - C. 34-38.

6. Абрамович Б.Н., Сычев Ю.А., Зимин Р.Ю. Оценка эффективности гибридных систем коррекции формы кривых тока и напряжения в электрических сетях с распределенной генерацией // Промышленная энергетика. - 2015. - № 8. - С. 49-53.

7. Abramovich B.N., Sychev Y.A. The control algorithm for active and hybrid correction systems of voltage and current waveforms // IEEE Conference 2016 Dynamics of Systems, Mechanisms and Machines. - Omsk, Russia, 15-17 November 2016. Article number: 7818962. DOI: 10.1109/Dynamics.2016.7818962.

8. Жежеленко И.В., Саенко Ю.Л. Показатели качества электроэнергии и их контроль на промышленных предприятиях. - М.: Энергоатомиздат, 2000. - 252 с

9. Железко Ю.С. Потери электроэнергии. Реактивная мощность Качество электроэнергии: Руководство для практических расчетов. - М.: ЭНАС, 2009. - 456 c.

10. Akagi H., Watanabe E.H., Aredes M. Instantaneous power theory and applications to power conditioning. 2 ed. - New Jersey: Wiley, IEEE Press, 2007. - $379 \mathrm{p}$.

11. Czarnecki L.S., Haley P.M. Unbalanced Power in Four-Wire Systems and its Reactive Compensation // IEEE Transactions on Power Delivery. - 2015. - V. 30. - № 1. - P. 53-63. D0I: 10.1109/TPWRD.2014.2314599.
3. За счет уменьшения падения напряжения на элементах ЭТК УЭЦН и снижения потребляемого тока установкой внедрение внутрискважинных компенсаторов реактивной мощности на нефтедобывающих объектах целесообразно с энергетической точки зрения.

12. Czarnecki L.S., Bhattarai P.D. A Method of Calculating LC Parameters of Balancing Compensators for AC Arc Furnaces // IEEE Transactions on Power Delivery. - 2017. - V. 32. - Iss. 2. P. 688-695. DOI: 10.1109/TPWRD.2016.2536681.

13. Sharon D. A Power factor definitions and power transfer quality in nonsinusoidal situations // IEEE Transactions on Instrumentation and Measurement. - 1996. - V. 45. - Iss. 3. - P. 728-733. DOI: $10.1109 / 19.494589$.

14. Sharon D. Reactive-power definitions and power-factor improvement in nonlinear systems // Proceedings of the Institution of Electrical Engineers. - 1973. - V. 120. - Iss. 6. - P. 704-706. DOI: 10.1049 /piee. 1973.0155

15. Копырин В.А., Смирнов 0.В. Оптимизация режимов потребления реактивной мощности установками электроцентробежных насосов // Известия Тульского государственного университета. Технические науки. - 2018. - № 2. - С. 450-458.

16. Копьрин В.А., Смирнов 0.В., Портнягин А.Л. Оценка энергетической эффективности использования внутрискважинных компенсаторов реактивной мощности / Омский научный вестник. - 2018. № 2 (158). - C. 78-83. DOI: 10.25206/1813-8225-2018-158-78-83.

17. Смирнов 0.В., Копырин В.А. К вопросу об использовании внутрискважинных компенсаторов реактивной мощности // Известия вузов. Нефть и газ. - 2015. - № 2. - С. 68-70.

18. Хамитов Р.Н., Охотникова А.А., Семеров Е.И. Причины выхода из строя силовых конденсаторов устройств компенсации реактивной мощности // Россия молодая: передовые технологии - в промышленности. - 2015. - № 1. - С. 278-282.

19. Кубарьков Ю.П., Голубева К.А., Макаров Я.В. Выбор мощности компенсирующих устройств для оптимизации уровней потерь в электрической сети // Вестник Самарского государственного технического университета. Серия: технические науки. - 2016. - № 4 (52). - С. 77-82.

20. Power loss reduction of $11 \mathrm{kV}$ feeder using capacitor banks to distribution transformers - a case study / V. Mishra, M.P. Sharma, B. Vyas, S.R. Ola // 2016 IEEE $1^{\text {st }}$ International Conference on Power Electronics, Intelligent Control and Energy Systems (ICPEICES). DOI: 10.1109/ICPEICES.2016.7853164.

21. Feasibility study of geothermal heat extraction from abandoned oil wells using a U-tube heat exchanger / S. Gharibi, E. Mortezazadeh, S.J. Hashemi Aghcheh Badi, V. Ali // Energy. - 2018. DOI: 10.1016/j.energy.2018.04.003.

22. Struchkov I.A., Rogachev M.K. The challenges of waxy oil production in a Russian oil field and laboratory investigations // Journal of Petroleum Science and Engineering. - 2018. DOI: 10.1016/j.petrol.2017.12.082.

Поступила 06.09.2018 г.

\section{Информация об авторах}

Konырин B.A., специалист отдела развития научных инициатив Тюменского индустриального университета.

Слирнов О.B., доктор технических наук, профессор кафедры электроэнергетики Тюменского индустриального университета.

Портнягин A.J., кандидат технических наук, директор Института геологии и нефтегазодобычи Тюменского индустриального университета.

Хамитов P.H., доктор технических наук, профессор кафедры электрической техники Омского государственного технического университета. 


\title{
INFLUENCE OF DOWNHOLE COMPENSATOR ON VOLTAGE DROP IN ELEMENTS OF A PRODUCTION WELL ELECTRICAL SYSTEM
}

\author{
Vladimir A. Kopyrin', \\ kopyrinva@gmail.com \\ Oleg V. Smirnov', \\ oleg_smirnov_1940@mail.ru \\ Alexey L. Portnyagin', \\ pal1979@mail.ru \\ Rustam N. Khamitov ${ }^{2}$, \\ apple_27@mail.ru \\ 1 Industrial University of Tyumen, \\ 38, Volodarsky Street, Tyumen, 625000, Russia. \\ 2 Omsk State Technical University, \\ 11, Mira Avenue, Omsk, 644050, Russia.
}

The relevance of the research is caused by the need to define the required voltage level of the supply transformer with regard to the downhole reactive power compensator, in order to prevent accidents in operation of electric centrifugal pumps' plants.

The aim of the research is to assess the change of voltage drop value in electrotechnical complex conductive elements of the electric center-pump plants for oil production using a downhole reactive power compensator.

The research object is the electrotechnical complex of electric center-pump plants for oil production.

Methods. Thesis of electrical engineering theoretical foundations, correct assumptions in preparation of mathematical models and computer simulation in the software package of symbolic mathematics Wolfram Mathematicain were used when developing the mathematical model of the electrotechnical complex of submersible plant for oil production.

Results. The authors have developed the equivalent circuit and mathematical model of electrotechnical complex plant of electric centerpump with a downhole reactive power compensator. The distribution of voltage drop values in the complex elements is shown using the high-quality vector diagram of voltage and current in the branches and nodes of the equivalent circuit. The analysis of the vector diagram showed that the in-hole compensator reduces the longitudinal and transverse components of the voltage drop, which positively affects the mode of operation of the oil production plant. The surface of the required voltage at the output of the supply transformer is obtained depending on submersible electric motor power value for different lengths and sections of the cable line. It was found that the use of the downhole compensator makes it possible to reduce the voltage drop in the current-conducting elements of the electrical engineering complex of the oil production plant to $50 \mathrm{~V}$.

\section{Key words:}

Downhole compensator, transformer soldering voltage, voltage drop, reactive power, circuit of substitution.

\section{REFERENCES}

1. Ivanovskiy V.N., Sabirov A.A., Dugowson A.V., Donskoy Yu.A., Bulat A.V., Zuev A.S., Yakimov S.B. Energy efficiency installations of electric centrifugal pumps. Equipment and technologies for oil and gas industry, 2016, no. 4, pp. 25-30. In Rus.

2. Bolovin E.V., Glazyrin A.S. Method for identifying parameters of submersible induction motors of electrical submersible pump units for oil production. Bulletin of the Tomsk Polytechnic University. Geo Assets Engineering, 2017, vol. 328, no. 1, pp. 123-131. In Rus.

3. Bukreev V.G., Sipaylova N.Yu., Sipailov V.A. Control strategy in accordance with economical criterion for electrotechnical installation of mechanized oil production. Bulletin of the Tomsk Polytechnic University. Geo Assets Engineering, 2017, vol. 328, no. 3, pp. 75-84. In Rus.

4. Nevostruev V.A. Operation's experience of energy-efficient ESP «Novomet». Engineering practice, 2017, no. 8, pp. 28-32. In Rus.

5. Yakimov S.B., Kaverin M.N., Tarasov V.P. About new prospects of the SEM application with the increased supply voltage for reduction of capital and operational expenses. Equipment and technologies for oil and gas complex, 2015, no. 4, pp. 34-38. In Rus.
6. Abramovich B.N., Sychev Y.A., Zimin R.Yu. Estimation of the efficiency of hybrid systems for correcting the shape of current and voltage curves in distributed distribution networks. Prom energy, 2015, no 8, pp. 49-53. In Rus.

7. Abramovich B.N., Sychev Y.A. The control algorithm for active and hybrid correction systems of voltage and current waveforms. IEEE Conference 2016 Dynamics of Systems, Mechanisms and Machines. Omsk, Russia, 15-17 November 2016. Article number: 7818962. DOI: 10.1109/Dynamics.2016.7818962.

8. Zhezhelenko I.V., Saenko Yu.L. Pokazateli kachestva elektroenergii i ikh control na promyshlennykh predpriyatiyakh [Indicators of power quality and their control in industrial enterprises]. Moscow, Energoatomizdat Publ., 2000. 252 p.

9. Zhelezko Yu.S. Poteri elektroenergii. Reaktivnaya moshchnost. Kachestvo elektroenergii: rukovodstvo dlya prakticheskikh raschetov [Loss of electricity. Reactive power. Quality of electricity: a guide for practical calculations]. Moscow, ENAS Publ., 2009. $456 \mathrm{p}$.

10. Akagi H., Watanabe E.H., Aredes M. Instantaneous power theory and applications to power conditioning. New Jersey, Wiley, IEEE Press, 2007. $379 \mathrm{p}$.

11. Czarnecki L.S., Haley P.M. Unbalanced Power in Four-Wire Systems and Its Reactive Compensation. IEEE Transactions on 
Power Delivery, 2015, vol. 30, no 1, pp. 53-63. D0I: 10.1109/TPWRD.2014.2314599.

12. Czarnecki L.S., Bhattarai P.D. A Method of Calculating LC Parameters of Balancing Compensators for AC Arc Furnaces. IEEE Transactions on Power Delivery, 2017, vol. 32, Iss. 2, pp. 688-695. D0I: 10.1109/TPWRD.2016.2536681.

13. Sharon D. A Power factor definitions and power transfer quality in nonsinusoidal situations. IEEE Transactions on Instrumentation and Measurement, 1996, vol. 45, Iss. 3, pp. 728-733. DOI: $10.1109 / 19.494589$.

14. Sharon D. Reactive-power definitions and power-factor improvement in nonlinear systems. Proceedings of the Institution of Electrical Engineers, 1973, vol. 120, Iss. 6, pp. 704-706. DOI: $10.1049 /$ piee.1973.0155

15. Kopyrin V.A., Smirnov 0.V. Optimization of reactive power consumption regimes by the electric centrifugal pumps installation. Bulletin of the Tula State University. Technical science, 2018, no. 2, pp. 450-458. In Rus.

16. Kopyrin V.A., Smirnov O.V., Portnyagin A.L. Estimation of the energy efficiency of the use of downhole compensators of reactive power. Omskiy nauchny vestnik, 2018, no. 2, pp. 78-83. DOI: 10.25206/1813-8225-2018-158-78-83. In Rus.

17. Smirnov 0.V., Kopyrin V.A. On the issue of using downhole reactive power compensators. Higher education institutions news. Neft i Gaz, 2015, no. 2, pp. 68-70. In Rus.
18. Khamitov R.N., Okhotnikova A.A., Semerov E.I. Reasons for failure of power capacitors for reactive power compensation devices. Russia is young: advanced technologies are in the industry, 2015, no. 1, pp. 278-282. In Rus.

19. Kubarkov Yu.P., Golubeva K.A., Makarov Ya.V. Selection of the power of compensating devices for optimization of losses in the electric network. Bulletin of the Samara State Technical University. Series: technical sciences, 2016, no. 4 (52), pp. 77-82. In Rus.

20. Mishra V., Sharma M.P., Vyas B., Ola S.R. Power loss reduction of $11 \mathrm{kV}$ feeder using capacitor banks to distribution transformers - a case study. 2016 IEEE $1^{\text {st }}$ International Conference on Power Electronics, Intelligent Control and Energy Systems (ICPEICES). DOI: 10.1109/ICPEICES.2016.7853164.

21. Gharibi S., Mortezazadeh E., Hashemi Aghcheh Badi S.J., Ali V. Feasibility study of geothermal heat extraction from abandoned oil wells using a U-tube heat exchanger. Energy, 2018. DOI: 10.1016/j.energy.2018.04.003.

22. Struchkov I.A., Rogachev M.K. The challenges of waxy oil production in a Russian oil field and laboratory investigations. Journal of Petroleum Science and Engineering, 2018. DOI: 10.1016/j.petrol.2017.12.082.

Received: 6 September 2018.

\section{Information about the authors}

Vladimir A. Kopyrin, specialist, Industrial University of Tyumen.

Oleg V. Smirnov, Dr. Sc., professor, Industrial University of Tyumen.

Alexey L. Portnyagin, Cand. Sc., director of the geology and gas-oil production institute of Industrial University of Tyumen.

Rustam N. Khamitov, Dr. Sc., professor, Omsk State Technical University. 